\title{
Inter-area frequency control reserve assessment regarding dynamics of cascading outages and blackouts
}

\author{
O. Alizadeh Mousavi*, M. Bozorg, R. Cherkaoui, M. Paolone
}

École Polytechnique Fédérale de Lausanne, 1015 Lausanne, Switzerland

\section{A R T I C L E I N F O}

\section{Article history:}

Received 21 February 2013

Received in revised form 13 August 2013

Accepted 1 October 2013

\section{Keywords:}

Cascading outages

Monte Carlo simulation

Interconnected power system

Short-term dynamics

\begin{abstract}
A B S T R A C T
The study of the dynamics taking place during power system blackouts is a subject that receives continuous attention in view of its inherent complexity and relevant consequences. Within this context, the paper aims at studying the role of the frequency control reserves (FCRs) on the cascading outages and the relevant short-term dynamics associated with the blackout mechanisms. The relationship between the large and small blackout frequency with respect to the value of FCRs is assessed. More in particular, the main contribution of this paper is to study the influence of the power system interconnections on its pre- and post-blackout behavior. For this investigation, a statistical procedure, based on the Monte Carlo simulation (MCS), is proposed. It performs a blackout risk analysis considering cascading outages as well as generators/loads response to the frequency deviation. The proposed procedure is then applied to the IEEE 118 bus system as an interconnected network characterized by three areas. The distributions and expected values of the blackout size are assessed for the whole system as well as for each area.
\end{abstract}

(c) 2013 Elsevier B.V. All rights reserved.

\section{Introduction}

Power transmission networks are large and complex systems that have experienced wide blackouts in the recent two decades (i.e. the Northeast and Italy blackouts in 2003 and India 2012). Although large cascading blackouts are relatively rare events, the investigation of their mechanism calls for significant efforts in view of the relevant consequences. Understanding the dynamics of power system components through their interactions with different control methods are the main challenges to comprehend a blackout mechanism. In this respect, several investigations have been performed in the literatures [1-6]. An additional aspect that increases the difficulty of the problem is the operation of single power systems within an interconnected continental-scale grid (e.g. interconnected networks in continental Europe - ENTSOE and North America - NERC). The areas of an interconnected power system generally profit from (i) increased security and (ii) mutual economically efficient generation. The higher security margins are a consequence of shared active power reserves. However, the security of the resulting interconnected power system could decrease with the increase of the interconnection and, consequently, with the increase of the power systems operation-complexity due to propagation of events, inter-area oscillations, etc. Hence, the delivery of required control actions should be carefully evaluated due

\footnotetext{
* Corresponding author. Tel.: +41 2169346 61; fax: +41 216934662 .

E-mail address: omid.alizadeh@epfl.ch (O. Alizadeh Mousavi).
}

to the counter-intuitive effects of opposing driving forces in power systems.

The analysis of the times series of blackouts size measures, e.g. energy not served (ENS) and load not served (LNS), in North America, China, Sweden, Norway, New Zealand, and continental Europe has shown a power law region in their distributions. This peculiarity demonstrates that the dynamics of blackouts can be associated to complex systems with self-organized criticality (SOC) feature [1]. In the system with the SOC characteristic, there are different types of variables with opposing driving forces that, in certain conditions, could drive the system into a critical operation state. In this case, after the occurrence of an initial fault or disturbance, cascading outages could cause a blackout (e.g. [2]). Furthermore, the power law region in the distributions implies that the blackouts of different scales may take place and the extreme events cannot be overlooked. The system may experience large blackouts with certain probabilities and the occurrences of small/large blackouts are not independent but correlated to each other (e.g. [3,7]).

It should be noted that the study of power systems short-term dynamics assumes a system with a fixed topology. Therefore, the continuous evolution of the power system state and configuration under complex dynamics are, in general, neglected [8]. By making use of the above-assumption, and within the context of risk assessment, this paper investigates the role of frequency control reserves (FCRs) on cascading outages and short-term dynamics of blackouts with particular reference to interconnected systems. The structure of the paper is the following: Section 2 provides the problem definition, Section 3 describes the blackout risk assessment methodology, Section 4 illustrates and discusses the simulation 
results with reference to the IEEE 118 bus test system considered as an interconnected network with three main areas, Section 5 discusses the possible approaches to solve the analyzed problem and Section 6 finally concludes the main outcomes of this study.

\section{Problem definition}

The different aspects of the cascading dynamics could be investigated in different time scales, namely, long-term, short-term, and transient dynamics [2]. The long-term dynamic investigates the role of load growth and engineering responses as external opposing forces to evaluate the system margins from critical loading in monthly or yearly time scales. The short-term dynamic in the range between several minutes to an hour, represents the internal system driving forces while the external forces are approximately remaining constant. It is associated with redistribution of power flows after the event and the response of controllers designed beforehand. The transient dynamics from milliseconds to seconds represents the inductive factor initiated by transient instability subsequent to large disturbances. The successive transient dynamics may cause abrupt outages.

The understanding of these dynamics can help to analyze characteristics of disasters and catastrophes, to evaluate the distance between the system's current state and its critical state and then to design preventive control strategies. In this respect, the blackout dynamics of the power grid should be appropriately modeled. Various methods have been proposed to model and analyze different aspects of blackouts in long-term, short-term and transient dynamics [9], such as, hidden failure model, OPA (ORNL-PSerc-Alaska) model, Manchester model, optimal power flow (OPF) based model and OPF transient stability (OTS) model. Besides these research models, several commercial tools have been developed in the industry, for instances, ASSESS, CAT, POM-PCM, TRELSS [9].

In this paper we focus specifically on the short-term dynamics of blackouts concerning the FCRs. The main idea is to study the dynamics of blackouts regarding the counteraction of the FCRs and the load shedding (including load curtailment and under-frequency load shedding) and their impacts on the cascading outages. In traditional approaches, regardless cascading outages, it is considered that the higher amount of the FCRs leads to the higher system security. Whereas, on one hand, a smaller amount of the FCRs may cause successive actions of under-frequency load shedding which increase the number of small blackouts. On the other hand, an excessive amount of the FCRs avoids the operation of the underfrequency load shedding and decreases the probability of small blackouts. However, it can increase the probability of line overloads that produces a triggering of cascading outages and consequent large blackouts. One may state that, within the context of the real operation of a given power system, the operator should ensure, for the different time frames, the transferability of FCRs avoiding transmission lines overloading. However, in the provision of control actions of interconnected power systems operated by several operators with limited coordination, it is practically impossible to take into account all possible contingency scenarios $N-k(k=1, \ldots, N)$ together with cascading failures.

In order to study the aforementioned phenomena, a blackout risk analysis method based on Monte Carlo simulation (MCS) is proposed. It takes into account different aspects such as the effects of cascading outages and the response of generating units/loads to the power imbalance. As a result, appropriate modeling of the fast and slow progress events, and the corresponding response of controllers, are necessary to study the blackout dynamics in power systems [10].

In blackout dynamics, cascading outages of overloaded components can progress either quickly or slowly. Fast progress events,

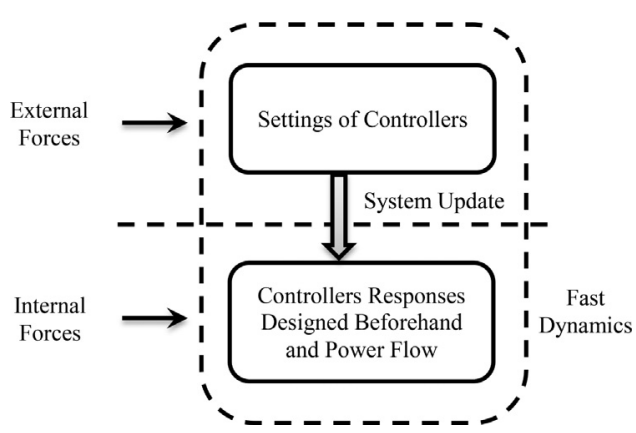

Fig. 1. Fast dynamics and external/internal forces.

initiated by large outages, involve different types of instability phenomena in time scales of seconds to several tens of seconds. Slow progress events are, typically, line tripping due to overloading in minutes time scales [11].

Concerning what FCRs entail, across different systems there are many different terms, definitions, and rules [12]. In this paper, the frequency reserve services are classified into automatically and manually activated FCRs given by $P_{g A}$ and $P_{g M}$, respectively. According to the hierarchical FCR classification (with primary, secondary and tertiary), the automatic FCR can be considered, in general, as primary and part of the secondary whereas the manual FCR is composed of the remaining part of the secondary and the tertiary. After a contingency occurrence, it is assumed that the automatic FCRs are decentrally activated in proportion to the frequency deviation to restore the equilibrium between generation and consumption in the operating time frame. Then, cascading outages progress in the system according to the re-established balance and the new power flows resulting from the activation of reserves. After a certain time, the system operator optimally deploys the automatic and manual FCRs to avoid the overloading and minimize the load curtailment in a centralized manner. Other control means, such as modification of line topology or adjustment of phase shifters, could also be used by the system operator. However they have been disregarded in the development of this paper for the sake of simplicity. In this respect, further investigations are needed.

In view of the above, the proposed model aims to effectively show the interaction between FCRs and load shedding as opposing forces in the short-term dynamics. The set points of the controllers designed beforehand, as external driving forces, are assumed to be fixed during fast dynamics. The general structure of this model is shown in Fig. 1.

From the practical point of view, the ENTSOE allows exchange, sharing and distribution of reserves between synchronous areas so that the activation of these reserves does not jeopardize the system security [13]. A survey study has been published in [6] concerning the assessment of inter-area FCR by means of different approaches that accounts for the system security. However, none of these approaches captures the dynamics of the cascading events and blackouts. Consequently the risk of large blackouts is not taken into consideration effectively.

\section{Blackout risk assessment method}

This section describes a statistical method which aims to numerically evaluate the risk of cascading blackouts regarding the FCRs. The method considers the following elements: (a) the effects of cascading outages due to overloads and hidden failure of protection systems, (b) the response of the FCRs of the generating units and the self-regulation of loads to power imbalance (i.e. frequency deviation) in each step of cascading outages. The preliminary version of this method is presented in previous works published by the 
authors in $[10,14]$. In comparison with these references, the method here presented for the blackout risk assessment is enriched by considering several mechanisms, such as random loading pattern, possibility of generating unit shut down in case of over-frequency and model of operators' corrective actions after several steps of cascading outages, as explained with more details at the end of Section 3. Beside, in contrary to Refs. $[10,14]$, the classification between the automatic and manual FCRs is taken into consideration. It must be noted that this model represents only some of the main important mechanisms associated to blackout dynamics. The other concomitant mechanisms, such as voltage excursions and collapse and transient instability, are not taken into consideration. Generally, these mechanisms do not impose high impact on the frequency control reserve and the frequency control procedure. That is due to the commonly acceptable assumptions to decouple the power systems analyses into (i) transient and steady state studies and (ii) voltage and frequency studies [15].

For the blackout risk assessment purpose, the MCS is applied to provide contingency scenarios including both generation and transmission outages. The system states are derived by sampling the state of each component based on its own availability. One of the merits of the MCS method is that it has the ability to look beyond the probable contingencies, taking into account rare, but significant, events. Moreover, the dagger sampling is used as a variance reduction technique to simulate the rare event cases and to improve the performance of the MCS [16]. This method is appropriate for two state variables and small probability events. In this sampling method, for each component with failure probability $p$, a single failure is randomly selected within each $1 / p$ trials. Hence, only one random number determines the state of the component for $1 / p$ trials [17]. Moreover, a random variation in the loading pattern is considered while the total load is kept constant. The reason for this is that the distribution of loads changes during the day and between days and cascading blackouts could start at different random loading patterns. The random loading pattern is modeled with a normal distribution whereas the mean is equal to the amounts of the loads. A $5 \%$ of the variance is also considered in the loading pattern distribution.

The contingency scenarios construct the system states and model the initial event. In the simulation procedure, after the initial event or after each step of cascading outages, there may be a power imbalance and, consequently, a frequency deviation in the system. It is assumed that the frequency deviation spreads uniformly in the system and all the generators with their automatic frequency control respond to this power imbalance. A distributed slack bus model is employed in such a way that all of the remaining dispatched generating units share the power imbalance according to their droop frequency-control characteristics with respect to their capacity limit $\left(\bar{P}_{g}=P_{g_{0}}+P_{g A}\right)$ where $P_{g_{0}}\left(\bar{P}_{g}\right)$ is the initial (maximum) power output of generators and $P_{g A}$ is the automatic FCR of generators. The self-regulation effect of loads is also taken into account. So, the frequency deviation $(\Delta f)$ of the system in each step of cascading outages is calculated according to the following equation [15]:

$$
\Delta f=\frac{-\Delta P}{\sum_{\Omega_{D}} D_{d}+\sum_{\Omega_{G}} 1 / R_{g}}
$$

where $\Delta P$ is the power imbalance in $M W, D_{d}$ and $R_{g}$ are the frequency characteristics of $d$ th load and gth generator (droop) in $M W / H z$ and $H z / M W$, respectively. $\Omega_{D}$ and $\Omega_{G}$ indicate the set of demands and generators, respectively.

It is assumed that, if the frequency deviation in the system exceeds or drops $5 \%$ of the nominal frequency, all the generators in this area trip and the system collapses [10]. Moreover, if the total minimum output of generators $\left(\sum P_{g}^{\min }\right)$ is higher than the total amount of load $\left(\sum P_{d}\right)$, the system is not any more controllable and it collapses.

Whenever the frequency deviation is in the allowed range, but the available capacities of the synchronized generating units are unable to satisfy the load, a frequency load shedding scheme uniformly disconnects the amount of the load to reach a new power balance. In the case of overfrequency in the allowed range, the generating units decrease their surplus output according to their frequency-control characteristics while accounting for their output lower bounds. After the generation and load balance is restored, a linearized load flow (DCLF) is applied to calculate the power flow and the transmission loading in each step of the cascading outages.

The outage of overloaded lines is one of the most important mechanisms in the power system blackouts. Moreover, the protection system has an undetected defect that remains dormant until an abnormal operating condition is reached. This state is often referred to as 'hidden failure'. In order to consider the effect of hidden failure in cascading outages, it is assumed that each transmission line has a different flow-dependent probability of incorrect trip. This characteristic is modeled as an increasing function of the line flow which is seen by the line protective relay [4]. As shown in Fig. 2, the probability of failure is small and equal to 0.01 for line flow lower than the line limit. This probability increases in proportion to the line flow between 1 and 1.4 times of the line limit. The lines that loaded more than 1.4 times of the line limit (overloaded lines) trips. It is worth to mention that in this model, in a given step of the cascading outages, the hidden failures can occur if none of the lines is loaded more than 1.4 times the capacity limit. Also, the lines which are connected to the last tripped lines are exposed to the hidden failure of protection system according to the obtained probability from Fig. 2.

As above-mentioned, after each step of cascading outage, power generation and consumption balance would be restored mainly through the generators automatic frequency response. These generating units reach their new operating points typically in tens of seconds. Therefore, the operator has the opportunity to implement some remedial (corrective) actions and minimize the amount of lost load, after several steps of cascading outages. The model of operators' response to contingencies is considered as a linearized optimal power flow (DC OPF) [10]. This control decision is calculated centrally for the interconnected system. Five steps of cascades are allowed before the operator intervention using DC OPF, which is an appropriate delay to model this action according to [18]. The aim of the DC OPF is the minimization of the lost load through re-dispatching the generating units and shedding some loads. It is assumed that in this step the operator can utilize both automatic and manual FCRs $\left(\bar{P}_{g}=P_{g_{0}}+P_{g_{A}}+P_{g_{M}}\right)$. Here, the generating unit shut down is also considered as a control action, whenever the total load $\left(\sum P_{d}\right)$ is less than the minimum generation output $\left(\sum P_{g}^{\min }\right)$.

In each step of the cascading outages, if the system is divided into multiple segments, the simulation would be separately performed for each segment. It is assumed that each segment continues its operation under this condition considering its own constraints.

For a given scenario $s$, the flowchart and models of the proposed blackout risk assessment procedure are given in Fig. 2. The stepby-step procedure to simulate the system for the sth scenario is as follows.

(1) Check the system split and perform the simulation for each segment.

(2) Calculate the frequency deviation due to contingency according to the generators automatic frequency control response and the load frequency characteristics in Eq. (1). 


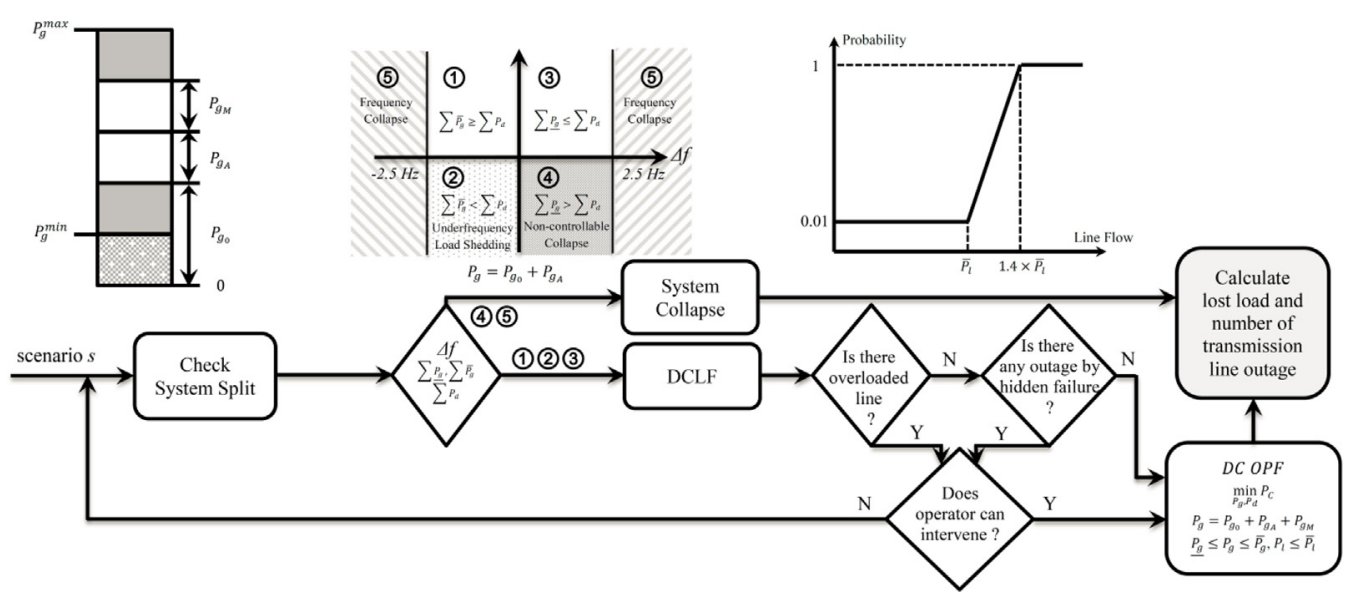

Fig. 2. Flowchart and models of the proposed simulation procedure for a given scenario s.

(3) According to the computed frequency deviation, demand, upper and lower limits of generators $\left(\overline{P_{g}}\right.$ and $\left.P_{g}\right)$, if the frequency deviation is higher than the frequency protection of the generating units (5) or the system is not controllable (4) ), the system collapses; otherwise (1)(2)(3) ) restore the generation and load balance and use a DCLF to find the transmission flows.

(4) If there is any overloading in the transmission system, trip the overloaded elements and then go to step (6); otherwise check the hidden failure of the protection system.

(5) If there is any outage due to hidden failure go to step (6); otherwise there is not any cascading effect in this scenario and go to step (7).

(6) Check if the operator can intervene and perform remedial (corrective) action; otherwise go to step (1). It is considered that the operator would have time to perform the remedial actions after five steps of cascading outages. This corrective action is a DC OPF for the minimization of lost load $\left(\min P_{C}\right)$ concerning the constraints of generators $\underline{P_{g}} \leq P_{g} \leq \bar{P}_{g}$ ) and transmission elements $\left(P_{l} \leq \bar{P}_{l}\right)$.

(7) Save the number of transmission line outage and the amount of lost load.

After simulating each scenario, the obtained amount of lost loads and the number of transmission component outages are used to evaluate the risk of blackout. For this purpose, the complementary cumulative distribution function (CCDF) of continuous random variables (lost load) is calculated by simply ranking the data and then scaling the ranked data. Also, the probability distribution function (PDF) of discrete random variables (number of transmission component outages) is calculated simply by assigning a probability to each possible value such that the total probability for all random variables is equal to 1 . In general, for the cumulative probability associated with a particular quantity (e.g. lost load), a sufficient number of the MCS samples should be calculated to ensure a specific level of accuracy $( \pm \delta)$ associated with a confidence level $(\sigma)$. The accuracy level is used as the stopping criteria for the MCS. For an output distribution, the regions closer to small size quantities will reach higher accuracy levels relatively faster compared to regions toward the heavy tail. The proposed method ensures the required level of accuracy associated with a value $x_{m}$ by determining what fraction of the samples fell at or above $x_{m}$. If so far we have had $n$ samples of MCS and $m$ have fallen at or above $x_{m}$, the cumulative percentile $\left(p_{m}\right)$ can be estimated as $p_{m}=m / n$. Then, by using Eq. (2) and monitoring $m$ and $n$ we can determine whether the required level of accuracy is obtained [16]. Here, the variable $x_{m}$ could be considered for the lost load as well as the number of outages.

$\delta=z \cdot \sqrt{\frac{p_{m} \cdot\left(1-p_{m}\right)}{n}}$

where $z$ is $1.96,2.56$ and 3.29 for $95 \%, 99 \%$ and $99.9 \%$ of the confidence level, respectively. Here, all the simulations come with $99 \%$ of the confidence level. In order to provide the stopping criteria for the MCS using Eq. (2), the value $x_{m}$ should be defined for any output quantity. Since in this paper we derive the CCDF of the lost load and the PDF of the outages number, two different values $x_{c}$ and $x_{p}$ are specified. In order to tune the process, $x_{c}$ and $x_{p}$ have been chosen according to a sensitivity analysis that accounts for the ratio of the lost load to the total load and the ratio of the number of outages to the total number of lines, respectively. With regard to $x_{c}$ and $x_{p}$, the distributions could be effectively demonstrated by obtaining an accuracy levels $(\delta)$ lower than 0.001 [14]. This value is selected by qualitatively analyzing the smoothness of distributions particularly in their tail behaviors. At the end of the MCS trials, the expected load not supplied (ELNS) is calculated as follows [17].

ELNS $=\frac{\sum_{n} P_{C}^{(n)}}{n}$

where $P_{C}^{(n)}$ is the lost load in $n$th trial of the MCS. It should be noted that the coefficient of variance $\left(c_{v}\right)$ of an expected value (e.g. ELNS) can be obtained according to

$c_{v}=\frac{\sqrt{\operatorname{var}(x)}}{E(x)}$

where $\operatorname{var}(\cdot)$ and $E(\cdot)$ are the variance and the expected value of the random variable $x$, respectively. When the MCS is employed to estimate an expected or a mean value, $c_{v}$ can be used as the stopping criteria [10]. Generally, fewer of the MCS trials are required to estimate an expected value (with a certain coefficient of variance) rather than a distribution function (with a certain accuracy level). The reason is that the stability of the expected values is not affected by the number of MCS trials as much as the smoothness of the tail behavior in the probability distributions.

\section{Simulations and discussions}

The aforementioned dynamics of blackouts in the FCRs is investigated with reference to the IEEE 118 bus system. As shown in Fig. 3, such a system is considered as an interconnected one with three main areas. The detailed data of generation, load, and transmission system are given in [19]. The unavailability of transmission 


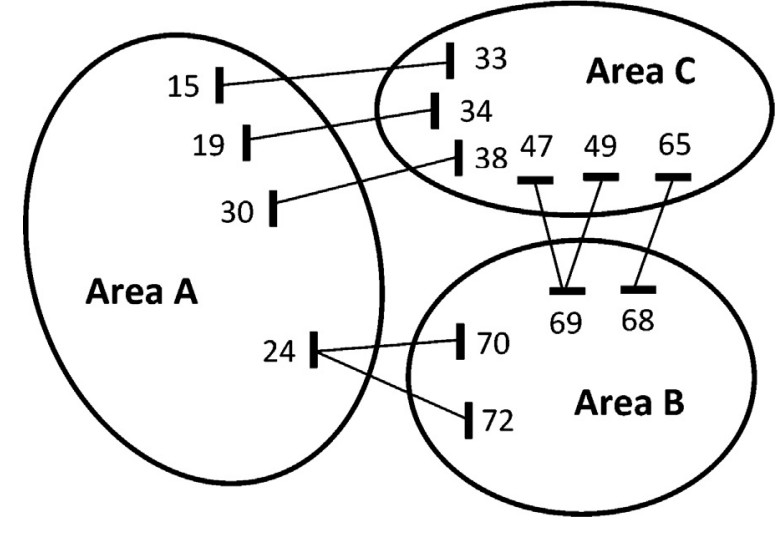

Fig. 3. IEEE 118 bus system with three areas.

lines $\left(U_{l}\right)$ and generation units $\left(U_{g}\right)$ are assumed equal to 0.01 . The response of generator $\left(r_{g}\right)$ and loads $\left(d_{n}\right)$ to the frequency deviation are considered equal to 0.05 and 1.00 , respectively. Note that $r_{g}$ and $d_{n}$ are in per-unit and they are converted to $R_{g}$ and $D_{n}$ for each generator and load by using Eq. (1).

The proposed blackout risk analysis method is applied for each area separately as well as for the interconnected system. Different amounts of the automatic reserve are specified for each one of the four study cases while the manual reserve is retained fixed. Then, the risks of blackout in each area and in the interconnected system are compared using the distribution of the lost load and the number of transmission component outages as the measures of the blackout size. For this purpose, the CCDF of the lost load data and the PDF

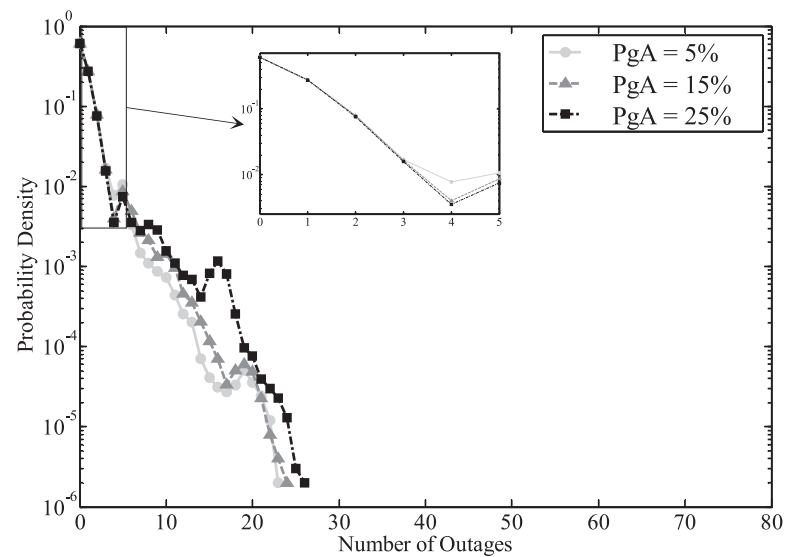

(a) Area A

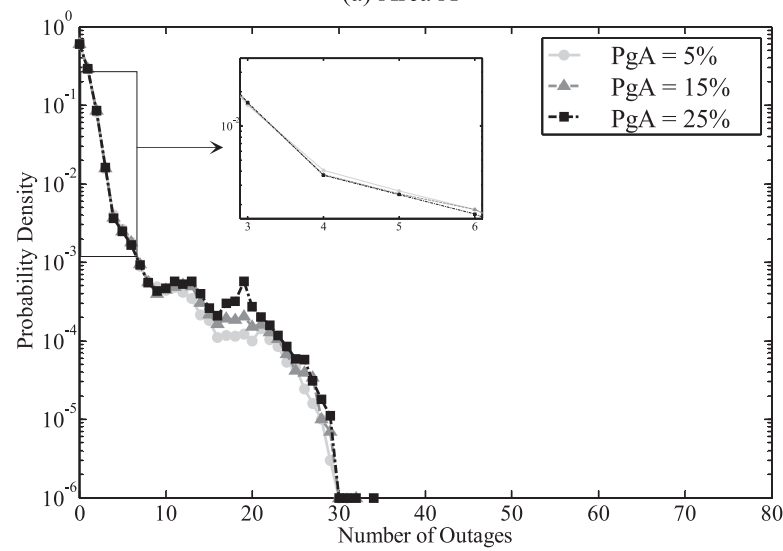

(c) Area C

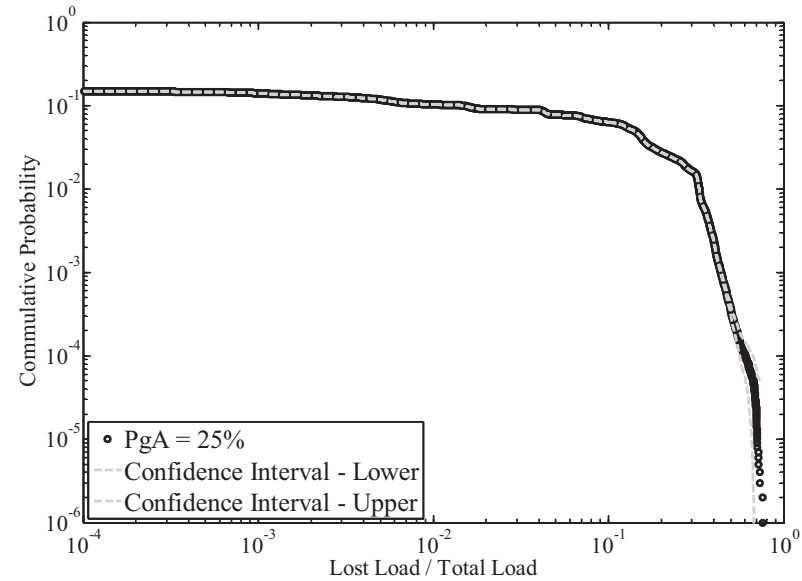

Fig. 4. $\mathrm{CCDF}$ of lost load for interconnected system for $R_{g A}=25 \%$ with its confidence interval.

of the number of transmission outages are calculated. The CCDF of the lost load and the PDF of the outage numbers are plotted in the log-log and log-linear axes, respectively, to effectively illustrate the distributions characteristics. For the convenience of comparing different systems, the amount of disconnected load divided by the total load, as a normalized blackout indicator, is used in this study.

The amount of generation and the maximum available reserve $\left(P_{g}^{\max }-P_{g 0}\right)$ for each area is given in Table 1 . In reality, different deterministic and probabilistic approaches can be used for the provision of the FCRs with respect to the security and economy

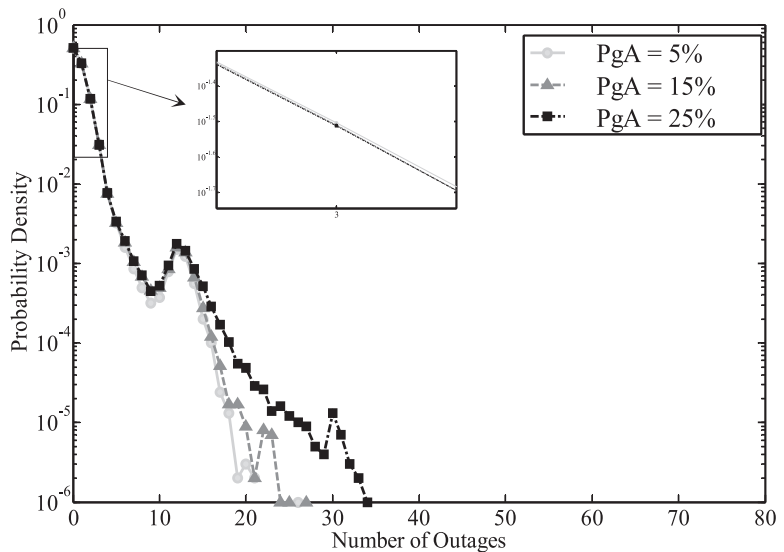

(b) Area B

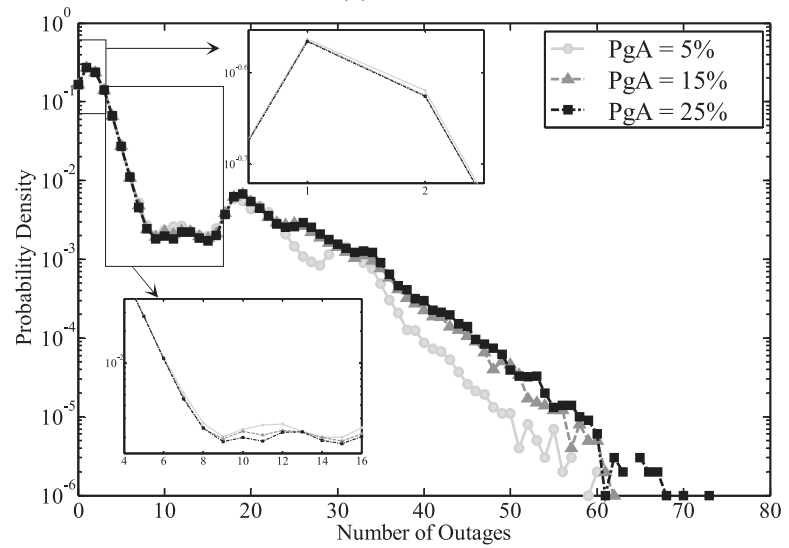

(d) Interconnected system

Fig. 5. PDF of number of transmission outages. 


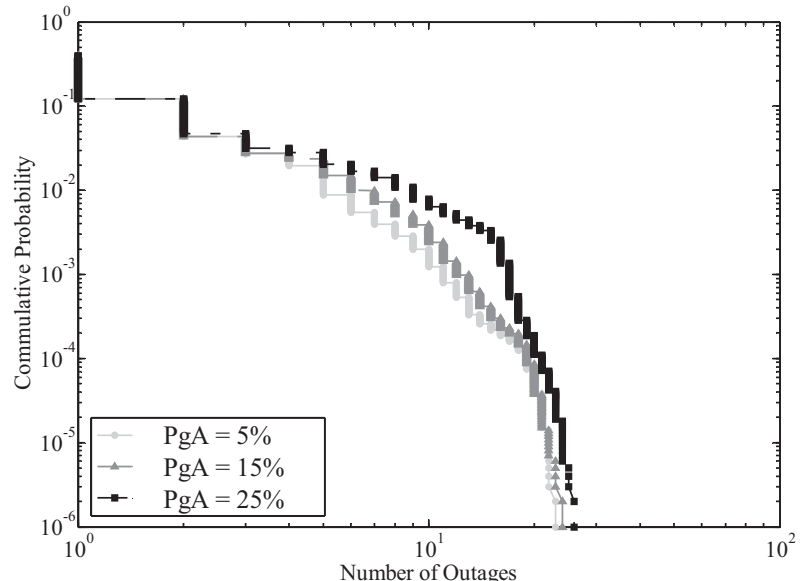

(a) Area $A$

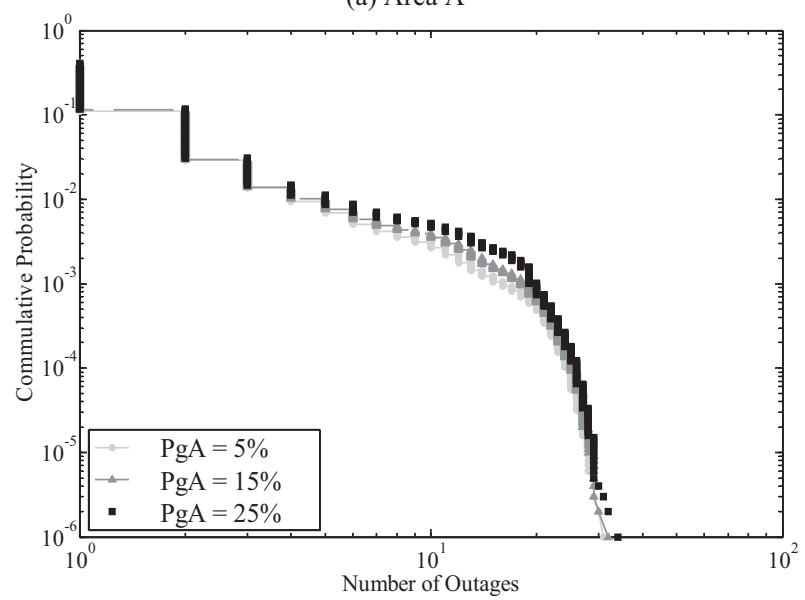

(c) Area $\mathrm{C}$

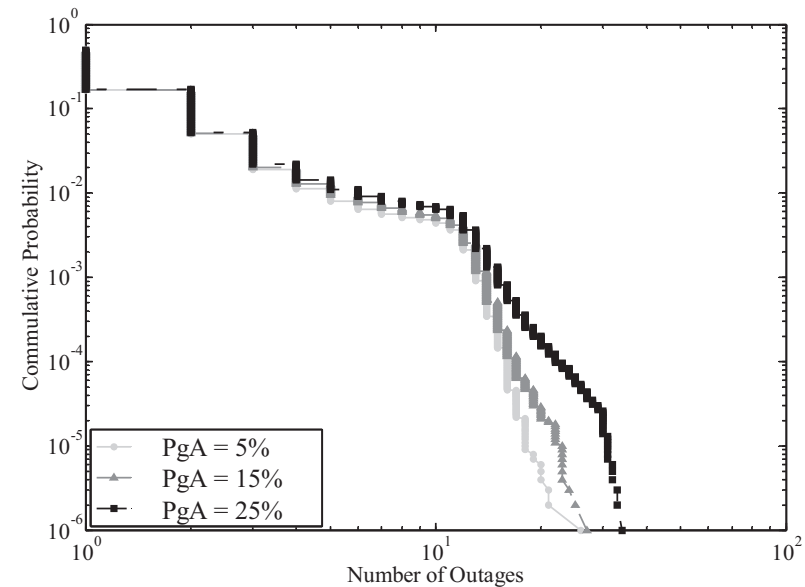

(b) Area B

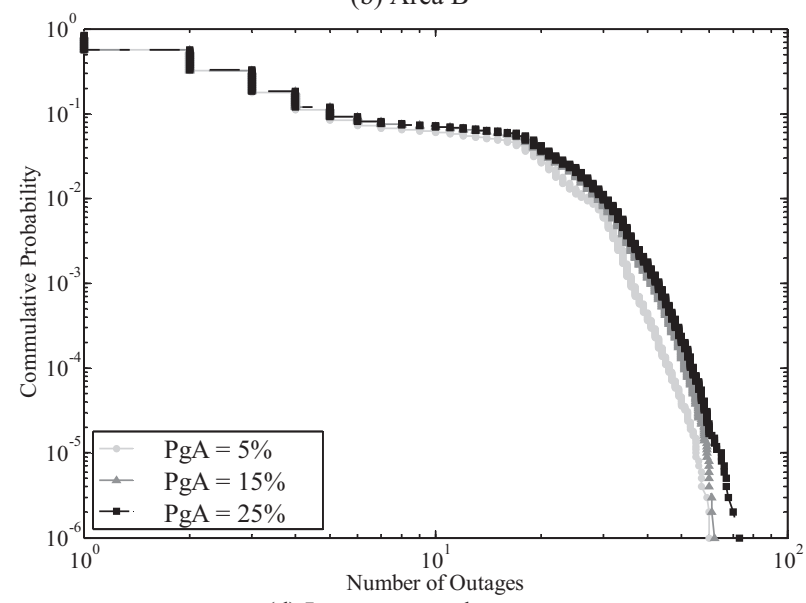

(d) Interconnected system

Fig. 6. CCDF of number of transmission outages.

Table 1

Generation and reserve of each area.

\begin{tabular}{llr}
\hline & Generation $(\mathrm{MW})$ & Maximum available reserve (MW) \\
\hline Area A & 1076.00 & 500.00 \\
Area B & 1866.53 & 1024.20 \\
Area C & 1547.84 & 700.00 \\
\hline
\end{tabular}

criterion. At the end, the total amount of FCRs could be represented as a percentage of the total capacity of generators. Moreover, it is assumed that the FCRs are evenly distributed based on the net generation and consumption and the remaining capacity of each generator. This simplifying assumption is somewhat conservative regarding the aim of this analysis because a given generator response to a frequency deviation will be provided, in reality, by different generators connected to the system. It reduces the risk of further overloads comparing to the case when a certain limited number of generators respond to the frequency deviation. Here, the simulations are performed for the four study cases with different amounts of automatic FCR (which are 5\%,15\% and 25\%) and with the same value of the manual FCR (which is 15\%). Since the manual reserve remains unchanged, from now whenever the term reserve is used, it refers to the automatic reserve.

It should be noted that, for each area, the neighboring ones beyond the interconnections, are modeled by a simple active power injection. In this way, each interconnection line is modeled by a consumption or generation.
As mentioned earlier, the obtained distributions from the MCS always come with a confidence interval which ensures the specified accuracy level. As an example, in the interconnected system case study with $R_{g A}=25 \%$, for the corresponding CCDF of the lost load, the confidence interval associated with the accuracy level is shown in Fig. 4. Another interesting point of the obtained results is that the maximum blackout size is 0.76 of the total load. This value is consistent with the reality, because no blackout has been recorded in which all the interconnected system demands have been interrupted. Additionally, since this work studies the shortterm dynamics of a power system with a fixed topology, it is expected to have an approximated power law region that covers a specific range of values. This region is much more limited in the distributions of each area because the system size is not sufficiently large.

The obtained results of the PDF of the number of outages for Area A, B, C and the interconnected system are given in Fig. 5a-d, respectively. These figures effectively demonstrate that the probabilities of small and large number of outages are dependent. Generally, as the amount of reserves increases, the probability of a higher number of outages increases. This behavior is more explicit in Area $B$ in comparison with the other ones. The reason is the higher amount of the reserve in Area B. However, the interconnection of the areas intensifies this behavior as depicted in Fig. $5 \mathrm{~d}$. Thus, in the interconnected system the provision of FCRs should receive more consideration.

Furthermore, the different regions in the CCDFs of the number of outages for different amounts of reserves concerning areas A, B, C 

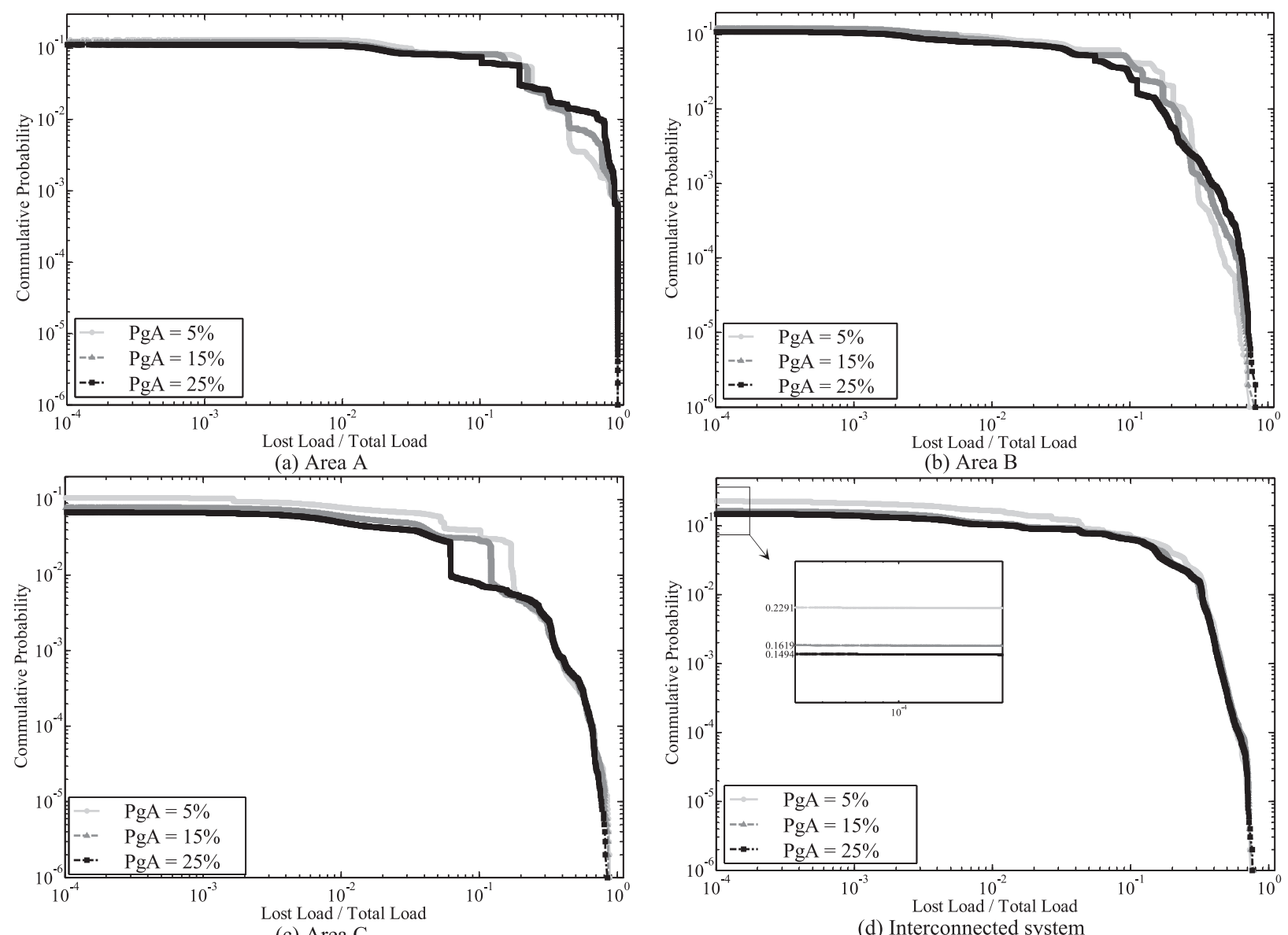

(c) Area C

Fig. 7. CCDF of lost load.

and the interconnected system, are presented in Fig. 6a-d, respectively. They indicate two regions for the small and large number of outages. The distribution curve with the lower values of reserve corresponds to the lower probability of a specified blackout size. According to Fig. 6, the increase of the reserve starting from $5 \%$ decreases (resp. increases) the probability of small (resp. large) number of outages. It means that the number of events with small (resp. large) number of outages decreases (resp. increases). This behavior becomes more significant in the interconnected system as illustrated in Fig. 5d. These results confirm the obtained results from the PDF of the number of outages.

The expected number of outages (ENO) is calculated analogous to Eq. (3) and the result for different study cases. Then, an unbiased expected number of outage (UENO) is defined as the ratio of the ENO to the total number of transmission lines in each study case. The value of UENO for every study case is given in Table 2. This table effectively demonstrates the increase of the UENO with the increase of the reserve. Also, for each amount of reserve, the value of UENO is higher in the interconnected system which demonstrates a higher level of risk. The CCDF of the lost load data for Area A, B, C and the interconnected system are given in Fig. 7a-d, respectively.

\section{Table 2}

Unbiased expected number of outages for all study cases for various amounts of reserve.

\begin{tabular}{rllll}
\hline$P_{g A}(\%)$ & Area A & Area B & Area C & Interconnected system \\
\hline 5 & 0.0129 & 0.0113 & 0.0114 & 0.0168 \\
15 & 0.0135 & 0.0115 & 0.0118 & 0.0181 \\
25 & 0.0147 & 0.0118 & 0.0121 & 0.0184 \\
\hline
\end{tabular}

Table 3

Probability of scenario with lost load for all study cases for various amounts of reserve.

\begin{tabular}{rllll}
\hline$P_{g A}(\%)$ & Area A & Area B & Area C & Interconnected system \\
\hline 5 & 0.1299 & 0.1227 & 0.1056 & 0.2291 \\
15 & 0.1196 & 0.1217 & 0.0784 & 0.1619 \\
25 & 0.1113 & 0.1103 & 0.0681 & 0.1494 \\
\hline
\end{tabular}

The CCDF of the lost load starts at different values. This value shows the total number of scenarios with lost load per total number of scenarios (probability of scenarios with lost load). For instance in Fig. $7 d$, the probability of scenarios with lost load considering the reserve $5 \%, 15 \%$ and $25 \%$ are $0.2291,0.1619$ and 0.1494 , respectively. These values for different cases are given in Table 3. In all the study cases, as illustrated in Fig. 7 and in Table 3, the higher amount of the reserve decreases the number of scenarios with lost load. It means that the higher reserve decreases the number of small blackouts. This specific aspect has been previously shown by the fact that higher a reserve increases the number of large blackouts.

In addition to studying the distributions of the blackout size, the expected measures (e.g. ELNS) could also be investigated. Hence, the ELNS for each case obtained from Eq. (3) is given in Table 4. The

Table 4

ELNS in every area and interconnected system for various amounts of reserves.

\begin{tabular}{rllll}
\hline$P_{g A}(\%)$ & Area A & Area B & Area C & Interconnected system \\
\hline 5 & 0.2550 & 0.2100 & 0.1322 & 0.8859 \\
15 & 0.2488 & 0.1719 & 0.0894 & 0.7048 \\
25 & 0.2600 & 0.1384 & 0.0637 & 0.6730 \\
\hline
\end{tabular}



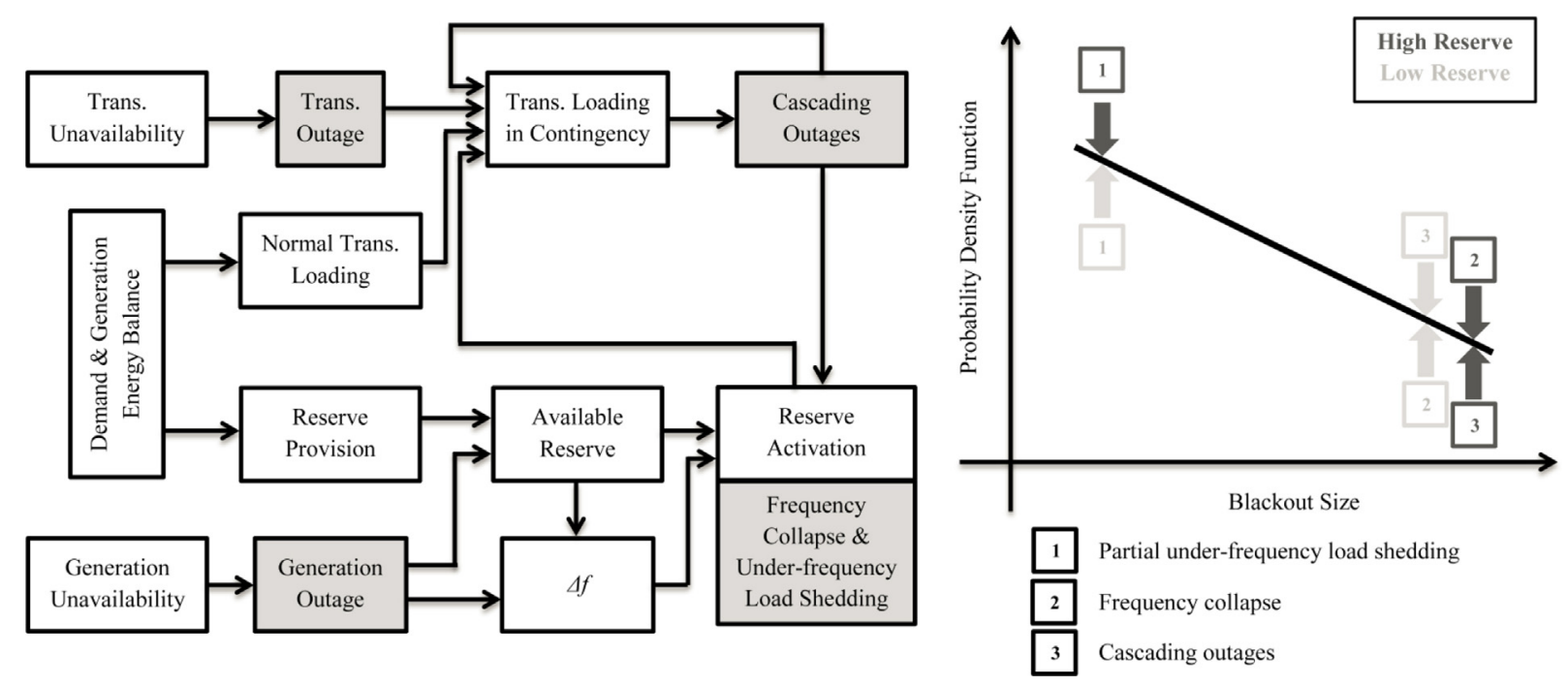

Fig. 8. (a) The system dynamics model for FCRs regarding cascading outages and (b) the effect of different forces on PDF of blackout size measure.

results show that the ELNS decreases by the increase of the reserve in all the cases as consistent to traditional findings. The expected values like ELNS cannot effectively reveal the impact of large blackouts since these events occur with small probabilities. As a matter of fact, this measure cannot show the impact of the aforementioned cascading dynamics with respect to the FCR. In order to evaluate the overall risk for blackouts of all possible scales, the unserved energy and its frequency should be known. The unserved energy can be derived as the product of the lost load and the duration of the blackout to obtain the direct cost. On one hand, the cost of small power interruptions, which are more frequent, have a strong impact on the total cost of power outages. On the other hand, if the costs of the large blackouts are considered, the risk of the large blackouts may exceed the risk of more frequent small blackouts [3]. Value at risk (VaR) and conditional value at risk (CVaR) are introduced to evaluate the blackout risk quantitatively with more focus on the large scale blackouts [20]. Non-linear indications are utilized to define a cost function in [3]. The discussions on the ELNS and the blackout cost are presented as general remarks and further details on these issues are out of the scope of this paper.

According to the results and discussions above, a system dynamics model for the FCRs considering the cascading outages is represented in Fig. 8a. As a complement, the effect of different forces on the PDF of the blackout size is given in Fig. 8b. As a result, the control of the system with such a sophisticated dynamic should be enhanced with sophisticated control or optimization approaches, specifically in the interconnected systems.

\section{Discussions for further investigations}

The effects of different policies of FCRs on the cascading outage, and the consequent risk of small and large blackouts, have been studied in this paper. As a result, a tradeoff should be assessed between large and small blackout frequency with respect to the value of FCRs, specifically in interconnected power systems. This new solution might affect both economical and reserve dispatching that would impose additional cost to the system operation. It should be noted that this paper faces the modeling, simulation and analysis of complex power grids; however, there are mathematical research challenges for the optimization of complex systems [21].

Alternatively, the qualitative indices $(Q I)$ method presented in [22] can also be proposed to manage the difficulties through a multimodal approach by introducing a threshold. As far as the QI indicates the normal condition, the current hierarchical control is effective. Whenever the QI indicates the system is departed from the normal condition, it informs the primary controllers whose logic has been changed to effectively act as conditions change. In this case, the resources should be adjusted to keep the interconnections operation as close as possible to the normal condition by means of hierarchical control, while critical desynchronization with the system islanding and load shedding could also be included. This approach is an online control that acts as a corrective action and it does not impose additional constraints on energy and reserve provision markets. This method could be implemented in a fully decentralized way or in the security control centers like Coreso (coordination of electricity system operators). However, the main issues are the definition of the $Q I$ and the value of the threshold to characterize the "extreme outlier events".

Furthermore, the fast power electronic controllers of FACTS and HVDC devices, installed on the interconnections, could be employed to control the flows between utilities during emergencies. They could act like firewalls against the spread of the cascading outages.

\section{Conclusions}

Based on the work presented by the authors in $[10,14]$, this manuscript proposes an improved method to estimate the blackout risk taking into account the classification of the automatic and manual FCRs, the random loading pattern, the possibility of generating unit shut down in case of over-frequency, and the model of operators' corrective actions after several steps of cascading outages.

The paper has investigated the dependency of the probability of different blackouts scales with respect to the FCRs. Within the context of the blackouts scales assessment, the first aim has referred to investigating the counter-intuitive effect of the under-frequency load shedding and the FCR in the short-term dynamics. Secondly, it is shown that this issue becomes more significant in interconnected power systems. Moreover, it is illustrated that the decrease of the amount of reserve increases the number of under-frequency load shedding and consequently increases the number of small blackouts. As a counter effect, the higher amounts of reserve increase the probability of overloading and cascading outages and consequently large blackouts.

The main conclusion of the paper is that this specific behavior should be considered in the reserve assessment of interconnected systems to prevent the risk of large blackouts. In particular, 
additional constraints should be taken into account in the operation of interconnected power systems in order to control the participation of each area in the reserve provision.

As the last remark, this paper only considers the provided reserve of the generators and the self-regulating effect of the loads; however, the required reserves could also be provided from the demand side. The fast demand side reserves contribute by curtailing a portion of the load. It effectively decreases the level of the illustrated complications, since it does not inject additional power into the grid after a disturbance. As a result, the probability of cascading outages and eventually large blackouts could decrease.

\section{Acknowledgments}

The paper takes into account the comments received during the presentation of a preliminary version at the 12th Probabilistic Method Applied to Power Systems Conference (PMAPS' 2012), Istanbul, Turkey, June, 10-14, 2012. Additionally, the authors thankfully acknowledge Swiss Electric Research as the results reported in this paper have been carried out within the framework of the research project "Security of Multi-Area Power Systems (MARS)".

\section{References}

[1] B.A. Carreras, D.E. Newman, I. Dobson, A.B. Poole, Evidence for self-organized criticality in a time series of electric power system blackouts, IEEE Trans. Circuits Syst. 51 (9) (2004) 1733-1740.

[2] M. ShengWei, X. AnCheng, Z. XueMin, On power system blackout modeling and analysis based on self-organized criticality, Sci. China Ser. E: Tech. Sci. 51 (2) (2008) 209-219.

[3] D.E. Newman, B.A. Carreras, V.E. Lynch, I. Dobson, Exploring complex systems aspects of blackout risk and mitigation, IEEE Trans. Reliab. 60 (1) (2011) 134-143.

[4] J. Chen, J.S. Thorp, I. Dobson, Cascading dynamics and mitigation assessment in power system disturbances via a hidden failure model, Elect. Power Energ. Syst. 27 (4) (2005) 318-326.
[5] I. Dobson, B.A. Carreras, D.E. Newman, A loading dependent model of probabilistic cascading failure, Probab. Eng. Inform. Sci. 19 (2005) 15-32.

[6] M.T. Schilling, R. Billinton, M.G. dos Santos, Bibliography on power systems probabilistic security analysis 1968-2008, Int. J. Emerg. Elect. Power Syst. 10 (3) (2009) 1-48.

[7] Initial review of methods for cascading failure analysis in electric power transmission systems, in: IEEE Power Eng. Soc. Gen. Meeting, Pittsburgh, PA, USA, July 2008.

[8] H. Ren, I. Dobson, B.A. Carreras, Long-term effect of the $n-1$ criterion on cascading line outages in an evolving power transmission grid, IEEE Trans. Power Syst. 23 (3) (2008) 1217-1225.

[9] M. Papic, et al., Survey of tools for risk assessment of cascading outages, in: IEEE Power Energ. Soc. Gen. Meeting, San Diego, 2011.

[10] O. Alizadeh Mousavi, R. Cherkaoui, M. Bozorg, Blackouts risk evaluation by Monte Carlo simulation regarding cascading outages and system frequency deviation, Electr. Power Syst. Res. 89 (2012) 157-164.

[11] M. Zima, G. Andersson, Wide area monitoring and control as a tool for mitigation of cascading failures, PMAPS (2004) 663-669.

[12] M. Milligan, et al., Operating reserves and wind power integration: an international comparison, Office of Energy Efficiency \& Renewable Energy, U.S. Dept. of Energ. Sl.: National Renewable Energy Laboratory (NREL), 2010.

[13] ENTSO-E Response, ACER Draft Framework Guidelines on Electricity Balancing Brussels, 2012 June 22

[14] O. Alizadeh Mousavi, M. Bozorg, R. Charkaoui, M. Paolone, Investigation of the blackouts complexity regarding spinning reserve and frequency control in interconnected power systems, PMAPS (2012) 982-987.

[15] P. Kundur, Power System Stability and Control, McGraw-Hill, India, 1994

[16] G. Rubino, B. Tuffin, Rare Event Simulation Using Monte Carlo Methods, John Wiley \& Sons, France, 2009.

[17] W. Li, Risk Assessment of Power Systems, Models, Methods, and Applications IEEE, Press, John Wiley \& Sons Inc., Canada, 2005.

[18] A. Bernstein, D. Bienstock, D. Hay, M. Uzunoglu, G. Zussman, Power Grid Vulnerability to Geographically Correlated Failures - Analysis and Control Implications, Technical Report, Columbia University, Electrical Engineering, 2011 November.

[19] Power systems test case archive [online]. Available: http://www.ee washington.edu/research/pstca/pf118/pg_tca118bus.htm

[20] S. Mei, X. Zhang, M. Cao, Power Grid Complexity, 1st ed., Springer and Tsinghua University Press, China, 2011.

[21] A Multifaceted Mathematical Approach for Complex Systems, Department of Energy Workshop, September 2011.

[22] M. Ilic, et al., Preventing future blackouts by means of enhanced electric power systems control: from complexity to order, Proc. IEEE 93 (11) (2005 1920-1941. 